\title{
Chest radiographic score and lactate dehydrogenase are independent risk factors linked to mortality in Middle East Respiratory Syndrome Coronavirus (MERS-CoV) patients
}

\author{
Karuna M. Das ${ }^{1 *}$ (D), Rajvir Singh², Khalid Al Dossari ${ }^{3}$, Sandeep Subramanya ${ }^{4}$, Shreesh Kumar Ojha ${ }^{5}$, \\ Taleb AlMansoori ${ }^{1}$ and Jamal Aldeen Alkoteesh ${ }^{6}$
}

\begin{abstract}
Background: Despite the dominance of Covid-19 in the current situation, MERS-CoV is found infrequently in the Middle East. When coupled with the chest radiographic score, serum biochemical parameters may be utilized to assess serum biochemical changes in individuals with different degrees of MERS-CoV infection and to predict death. The purpose of this study was to examine the association between increased LDH levels and severe MERS-CoV outcomes utilizing ventilation days and an elevated chest radiographic score.

Results: Fifty-seven patients were included in the retrospective cohort. The mean age was $44.9 \pm 13.5$ years, while the range was between 12 and 73 years. With an average age of $53.3 \pm 16.5$ years, 18 of 57 (31.6\%) patients were classified as deceased. The deceased group showed a substantially greater amount of LDH than the recovery group ( $280.18 \pm 150.79$ vs. $1241.72 \pm 1327.77, p=0.007$ ). A cut-off value of $>512 \mathrm{LDH}$ was established with a C-statistic of 0.96 (95\% Cl 0.92-1.00) and was $94 \%$ sensitive and $93 \%$ specific for mortality. Multivariate cox regression analysis revealed that $\log _{\mathrm{e}}(\mathrm{LDH})$ (adjusted HR: 9.91, 95\% Cl: 2.44-40.3, $p=0.001$ ) and chest radiographic score (adjusted HR: $1.24,95 \%$ Cl: $1.05-1.47, p=0.01$ ) were risk factors for mortality, whereas ventilation days were a protective factor (adjusted HR: 0.84, 95\% Cl: 0.76-0.93, $p=0.001$ ).
\end{abstract}

Conclusion: According to our results, blood LDH levels of $>512$ had a $94 \%$ sensitivity and $93 \%$ specificity for predicting in-hospital mortality in patients infected with MERS-CoV. The chest radiographic score of $11.34 \pm 5.4$ was the risk factor for the mortality (adjusted Hazard ratio HR: 1.24, 95\% Cl: 1.05-1.47, $p=0.01$ ). Thus, threshold may aid in the identification of individuals with MERS-CoV infection who die in hospital.

Keywords: MERS-COV, ARDS, LDH, Mortality, Prediction

\section{Background}

Global scientific interest has focused on the Coronavirus 2 Severe Respiratory Syndrome because of the current epidemic (SARS-CoV-2). An earlier zoonotic and extremely dangerous coronavirus, the Middle East

\footnotetext{
${ }^{*}$ Correspondence: daskmoy@gmail.com

1 Department of Radiology, College of Medicine and Health Sciences, UAEU, 17666 Al Ain, United Arab Emirates

Full list of author information is available at the end of the article
}

Respiratory Syndrome coronavirus (MERS-CoV), is a source of worry, particularly in Saudi Arabia and surrounding nations. MERS-CoV epidemics are associated with a death rate of up to $34 \%[1,2]$. Until recently, 2586 MERS-CoV cases have been reported globally, with 939 fatalities [3]. Since January 2021, Saudi Arabia has recorded five MERS-CoV cases, including three fatalities [3]. Pneumonia is often present at the time of presentation, and severe infection develops, particularly in 
elderly patients with comorbidities [4]. Various studies have shown the diagnostic use of chest radiography, particularly radiographic scores for clinical correlation in viral lung diseases such as SARS-CoV-1 and MERS-CoV [5-7].

Primary cases have a $35 \%$ mortality rate, whereas secondary cases have a $20 \%$ mortality rate [4]. An elderly patient, male predominance, diabetes mellitus, chronic lung disease, chronic renal illness, low albumin level, and progressive lymphocytopenia were all indicators of a grim prognosis [8-10]. Lactate dehydrogenase (LDH) is found extracellularly in response to cell injury or inflammation [11]. LDH is a biomarker associated with individuals with the poorest prognosis when infected with viruses [11-13]. Elevated serum LDH levels are associated with several respiratory diseases, including CAP (Community-acquired pneumonia), Pneumocystis Jiroveci pneumonia (PJP), and Mycoplasma pneumonia [4, 13]. LDH levels were increased in individuals with active MERS$\mathrm{CoV}$ infection and lung fibrosis associated with MERS $[14,15]$. Although patients with severe MERS-CoV had increased serum LDH levels, no research has examined the effect on the disease's severity or death [15]. Thus, a better knowledge of the function of LDH and the relationship between the chest radiographic score and other parameters could aid in treating MERS-CoV patients. This retrospective study determines the LDH level and its correlation with chest radiographic score and their clinical significance for MERS-CoV infection severity and mortality in a Middle Eastern population.

\section{Methods}

\section{Data sources and the study population}

The study was authorized by the institutional review boards (IRBs) and ethics committees. Because of the retrospective nature of this research, formal informed consent was not needed. All investigations adhered to the Helsinki Declaration.

The retrospective research included 57 MERS-CoV patients with accessible chest radiographs and laboratory data who were verified by RT-PCR [16]. Between July 1, 2014, and September 13, 2019, the researcher collected 57 patients diagnosed with MERS-CoV from two separate hospitals in the Middle East. The following were the inclusion criteria: (1) serum LDH levels, and (2) acceptable chest radiographs. Those with deficient chest radiographs and lack of serum LDH levels were excluded. All medical records were surveyed, and data were abstracted before being recorded into and crosschecked in a computerized database. In this research, the investigators examined independently predicted factors, which were categorized: (1) the chest radiographic score (2) days of ventilation (3) diabetes mellitus and its
Association (4) Patients' ages; (5) Gender; and (6) Laboratory values for lactate dehydrogenase (LDH), alanine transaminase (ALT), and aspartate aminotransferase (AST) that were associated with the patient's mortality. Separately, two thoracic radiologists with 12 (KD) and 22 (K.M.D) years of expertise in thoracic imaging evaluated the anonymized chest radiographs on clinical picture archive and communication system monitors. They graded the opacification of the lung parenchyma based on chest radiographs. Each lung was divided into three zones, and the extent to which each zone was involved was determined [5]. On a scale of 0 (normal) to 4 (complete involvement of one lung zone), the air space opacities caused by MERS-CoV lesions were evaluated. The results for each of the six zones in each chest radiograph sample were averaged to provide a composite chest radiographic score ranging from 0 to 24 according to the degree of lung parenchymal involvement. Disputes between the two radiologists were resolved via an agreement. Patients were divided into two groups: those who died were classed as the deceased, while those who survived were categorized as the recovery group.

\section{Statistical analysis}

For interval variables such as age, ALT, and AST, descriptive statistics as means and standard deviations were calculated, while for categorical variables, the frequency was calculated using percentages. Student $t$ tests were used to determine the significance of the difference between the mean levels of two groups ( $\mathrm{LDH} \leq 512 \mathrm{vs.} \mathrm{LDH}>512)$ for interval variables, with $p$-values for equal or unequal variance according to Levene's test. The connection between the two groups and categorical variables such as gender, diabetes mellitus, and deceased, and abnormal chest $\mathrm{x}$-ray was examined using chi-square or Fisher's exact tests. The correlation coefficient between the chest radiography score and the ventilation days was determined. The Kaplan-Meier survival curve was shown as a graph representing the median survival time in days. After adjusting for relevant and crucial factors, a multivariate Cox proportional hazard analysis was conducted to determine the relevance of $\log _{e}(\mathrm{LDH})$. Sensitivity and specificity were computed using c- statistics to determine the discriminatory ability of the LDH cut-off value. A $p$-value of 0.05 was considered statistically significant (two-tailed). SPSS 26.0 statistical software was used to perform the analysis.

\section{Results}

The study population comprised 57 confirmed cases of MERS-CoV, ranging in age from 44.9 to 13.5 years and with a male to female ratio of 24:33. Eighteen of 57 (31.6\%) patients died, with an average age of $53.3 \pm 16.5$ years, 
and 39 of $57(68.4 \%)$ patients recovered, with an average age of $41.1 \pm 9.9$ years $(p=0.001)$. When compared to the recovery group, the deceased group exhibited substantially higher LDH levels $(280.18 \pm 150.79$ vs. $1241.72 \pm 1327.77, p=0.007)$ and concurrently increased AST $(56.7 \pm 59.6$ vs. $442.2 \pm 1076.1, p=0.14)$ and ALT $(65.2 \pm 561.6$ vs. $124.6 \pm 174.8, p=0.18)$. Compared to the usual value, the AST and ALT were increased by $8 \times U L N$ and $3 x U L N$, respectively (ULN $=$ upper limit of normal). Thirty-eight patients out of 57 required ICU hospitalization, with 32 needing mechanical ventilation. The median survival time was 21 days (Fig. 1). The follow-up time ranged from one day to 210 days.

The data were then examined further using the cut-off value for the LDH variable (Fig. 2). The $>512 \mathrm{LDH}$ level $(\mathrm{LDH} \leq 512$ and $\mathrm{LDH}>512$ was chosen at a C-statistic of 0.96 , 95\% CI: $0.92-1.00$ ) was found to be $94 \%$ sensitive and $93 \%$ specific for mortality. The cut-off value of the $>512 \mathrm{LDH}$ was found to be associated with the deceased group [1 (2.7\%) vs. $17(85.0 \%), p=0.001)]$. The $\mathrm{LDH}>512$ group had a higher proportion of age in years, ventilation days, chest radiographic score, abnormal chest $x$-ray, and diabetes mellitus than the $\leq 512$ LDH level group (Tables 1 and 2, Figs. 3, 4). Correlations between chest radiographic score and ventilation days were statistically significant $(r=0.44, p=0.001)$.

Age in years, gender, ALT, AST, ventilation days, chest radiographic score, diabetes mellitus, and $\log _{\mathrm{e}}$ of LDH (Log transformation of LDH levels were done because of skew data) were used as independent variables and deceased as the dependent variable with admission days as a time factor variable in multivariate Cox regression analysis. The analysis revealed that ventilation days were a protective factor (adjusted HR: 0.84, 95\% CI: $0.76-0.93, p=0.001$ ), while the chest radiographic score (adjusted Hazard ratio HR: 1.24, 95\% CI: 1.05-1.47, $p=0.01$ ) and $\log _{\mathrm{e}}(\mathrm{LDH})$ (adjusted HR:

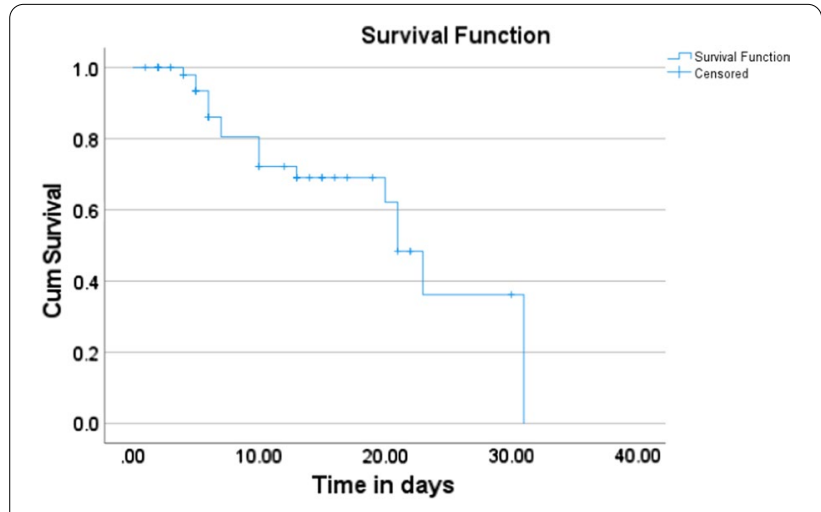

Fig. 1 Survival curve for 57 cases who are having LDH values

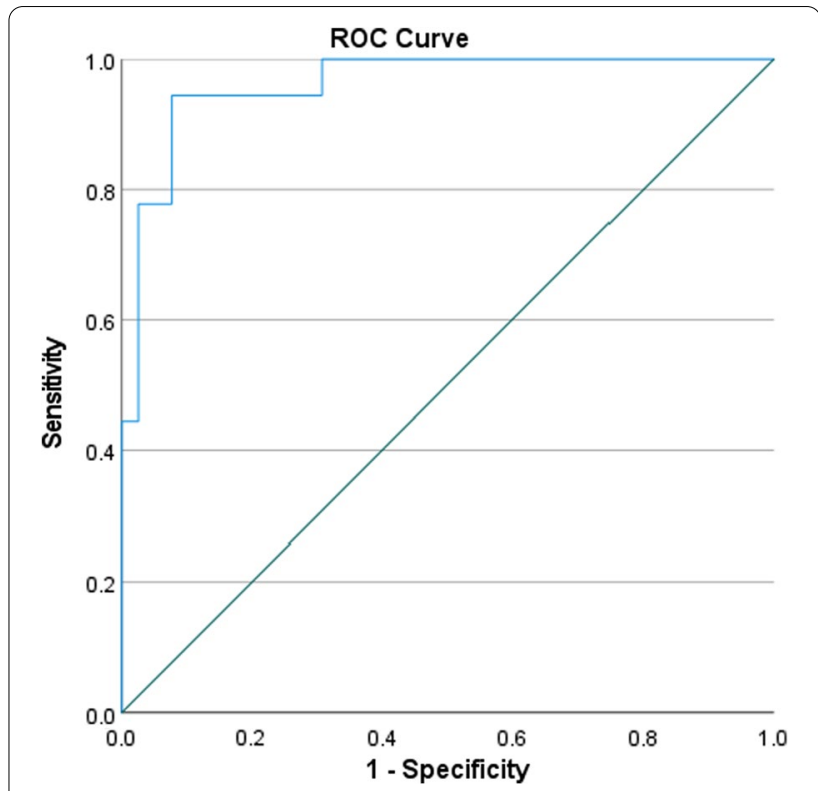

Fig. 2 ROC curve to decide LDH level to discriminate between deceased versus recovered. It shows LDH cut-off value $>512$ had $94 \%$ sensitivity and $93 \%$ specificity for the mortality showing the cut-off > 512 LDH value discriminate accurately 96\% (95\% Cl: 92\% to $100 \%$ ) between deceased and recovered patients

9.91, 95\% CI: 2.44-40.3, $p=0.001)$ were risk factors for mortality (Fig. 2). There was no significant relationship between ALT, AST, age, or gender, and mortality in this investigation (Table 3).

Table 1 Comparison of clinical parameters according to LDH level in 57 MERS-Cov patients

\begin{tabular}{llllll}
\hline Variable & \multicolumn{6}{l}{ Group statistics } \\
\cline { 2 - 6 } & LDH & $\boldsymbol{N}$ & Mean & Std. Deviation & $\boldsymbol{p}$-value \\
\hline AST & $\leq 512$ & 37 & 45.43 & 41.51 & 0.11 \\
& $>512$ & 20 & 424.55 & 1018.84 & \\
ALT & $\leq 512$ & 37 & 54.22 & 49.58 & 0.04 \\
& $>512$ & 20 & 138.95 & 166.31 & \\
Age in years & $\leq 512$ & 37 & 41.3 & 11.25 & 0.01 \\
Ventilation days & $>512$ & 20 & 51.6 & 14.97 & \\
& $\leq 512$ & 37 & 2.81 & 6.01 & 0.001 \\
Chest radiographic & $>512$ & 20 & 11.8 & 9.56 & \\
score & $\leq 512$ & 37 & 2.01 & 3.7 & 0.001 \\
Admission days & $>512$ & 20 & 11.34 & 5.43 & \\
& $\leq 512$ & 37 & 9.22 & 6.85 & 0.05 \\
& $>512$ & 20 & 13.55 & 9.08 & \\
\hline
\end{tabular}


Table 2 Association of demographic and clinical characteristics according LDH in 57 MERS-Cov patients

\begin{tabular}{llll}
\hline Variable & LDH $\leq \mathbf{5 1 2}(\boldsymbol{n}=\mathbf{3 7})$ & $\mathbf{L D H}>\mathbf{5 1 2}(\boldsymbol{n = 2 0 )}$ & $\boldsymbol{p}$-value \\
\hline Male & $17(45.9 \%)$ & $7(35 \%)$ & 0.42 \\
Deceased & $1(2.7 \%)$ & $17(85.0 \%)$ & 0.001 \\
$\begin{array}{ll}\text { Chest X-ray Abnor- } \\
\text { mal }\end{array}$ & $21(56.8 \%)$ & $20(100 \%)$ & 0.001 \\
Diabetes mellitus & $1(2.7 \%)$ & $9(45.0 \%)$ & 0.001 \\
\hline
\end{tabular}

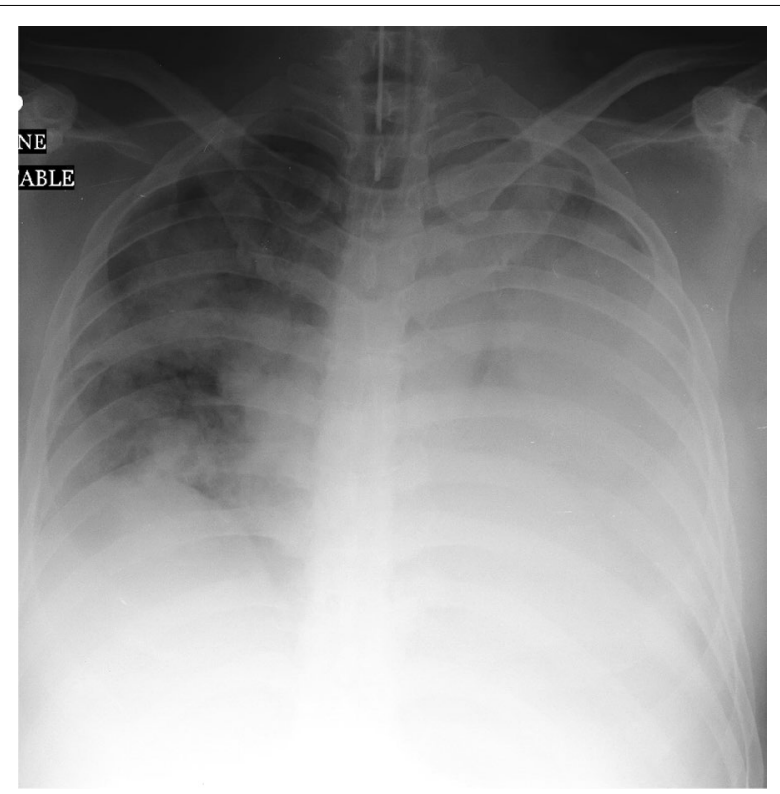

Fig. 3 A 52-year-old man who developed Middle East respiratory syndrome coronavirus. Frontal chest radiograph obtained on day 8 shows bilateral multifocal patchy airspace disease with predominant hemiopacification of the left lung; chest radiographic score is 14 with $\mathrm{LDH}$ of 760 . The patient died ten days after initial admission

\section{Discussion}

MERS-Cov spread across the Middle East, causing human illness via solitary cases, community clusters, and nosocomial outbreaks. This retrospective research showed a link between an elevated serum LDH level and death in MERS-CoV patients. According to ROC analysis, serum LDH levels of $>512$ throughout the illness were $94 \%$ sensitive and $93 \%$ specific for death and were linked with the deceased group [1 (2.7\%) vs. 17 (85.0\%), $p=0.001)]$. The Cox proportional hazard model showed that increased serum $\log _{\mathrm{e}}(\mathrm{LDH})$ levels during admission days were an independent predictor of MERS-CoV severity and mortality (adjusted HR: 9.91, 95\% CI: 2.44-40.3, $p=0.001$ ). We observed that individuals with higher chest radiographic scores (adjusted HR: 1.24, 95\% CI: $1.05-1.47, p=0.01$ ) were more likely to have a fatal outcome, as shown by the Cox proportional hazards model.

Although deceased individuals had a significantly higher mean age $(54.37 \pm 16.68, p=0.001)$, age was not a risk factor after adjusting for other multivariate cox regression analysis factors (adjusted HR: 0.99, 95\% CI: $0.95-1.03 ; p=0.60$ ). Our present observation was contrary to previous experience that older age is associated with increased risk of mortality in MERS-CoV patients [17]. One reason our deceased cohort's mean age was lower $(54.37 \pm 16.68, p=0.001)$ than in previous series with higher range of mortality [17]. Ahmed et al. found that patients aged $\geq 60$ years had a significantly higher death rate (45.2 percent vs 20.0 percent; $p=0.001$ ) when compared to patients aged $<60$ years [18]. Diabetes mellitus (adjusted HR: 1.01, 95\% CI: 0.24-4.24, $p=0.99$ ) and chest radiographic score (adjusted HR: 1.24, 95\% CI: $1.05-1.47, p=0.01$ ) were risk factors that could be interpreted as increasing the likelihood that the problem will deteriorate further and exacerbate the burden of the current condition. Similarly, after adjusting for other relevant factors, it was found that LN (Log Natural) LDH was a very significant risk factor for mortality (adjusted HR: 9.91, 95\% CI: 2.44-40.30, $p=0.001)$. Increased ventilation days are rated as protective in multivariate cox regression analysis (adjusted HR: 0.84, 95\% CI: 0.76$0.93, p=0.001$ ), which can be interpreted to mean that an increase in ventilation days may confer resilience to patients undergoing critical care, decreasing the likelihood of a fatal outcome.

According to Assiri et al., 49\% of their MERS-CoV patients exhibited increased LDH levels, compared to $50 \%-71 \%$ of global SARS-CoV-1 cases; however, no particular level was noted in MERS-CoV patients who died [15]. Ghamadi et al. found that 62.7 percent of their MERS-CoV patient groups had increased $\mathrm{LDH}>300 \mathrm{U} / \mathrm{L}$, with 56.3 percent surviving and $43 \%$ dying [19]. They have not referred to a cut-off point

\footnotetext{
(See figure on next page.)
}

Fig. 4 A 68-year-old man with Middle East respiratory syndrome coronavirus. Serial chest radiographs show a type 4 pattern of progression. A A frontal chest radiograph shows airspace disease involving the in the right upper, mid and lower zone with a chest radiographic score of 12 with bilateral pleural effusion. B On day 4, a follow-up frontal chest radiograph shows further deterioration of right lung air space opacity with increase of bilateral pleural effusion. C On day 6 , further worsening of the bilateral air space opacities noted. D A CT scan done on the same day shows bilateral pleural effusion with bilateral air space consolidations. $\mathbf{E}$ On day 8 , the patient further deteriorated and was put on ventilation. At this stage his serum LDH was 1326 and he died on day 10 
(a)

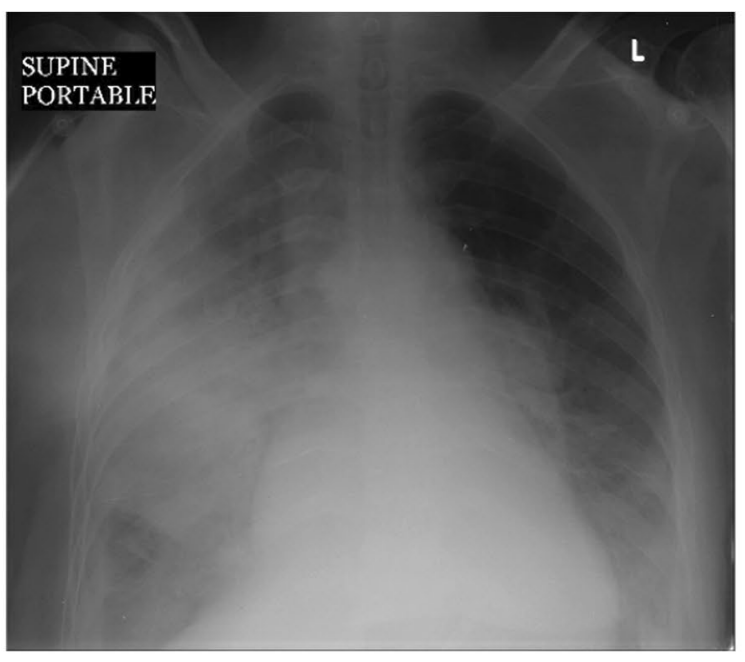

(b)

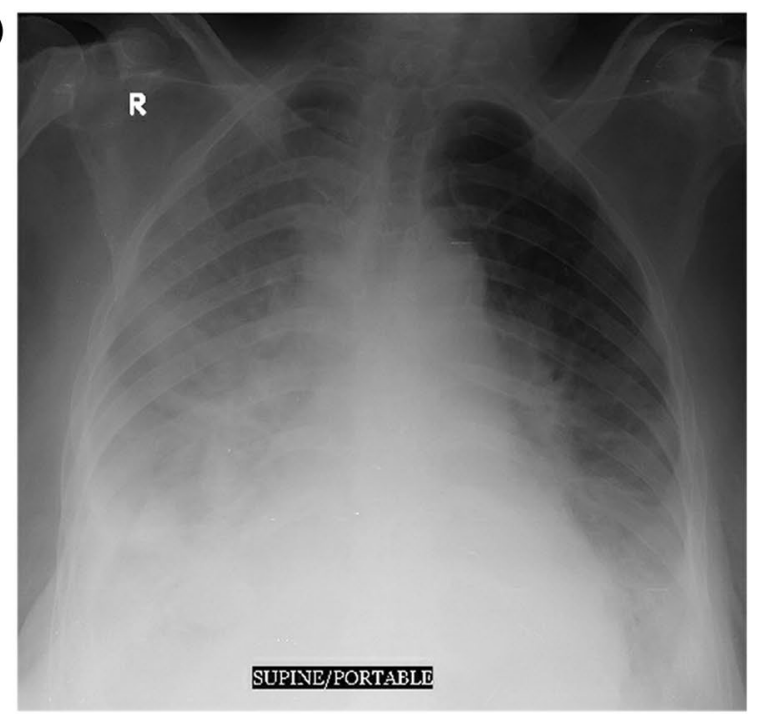

(e)

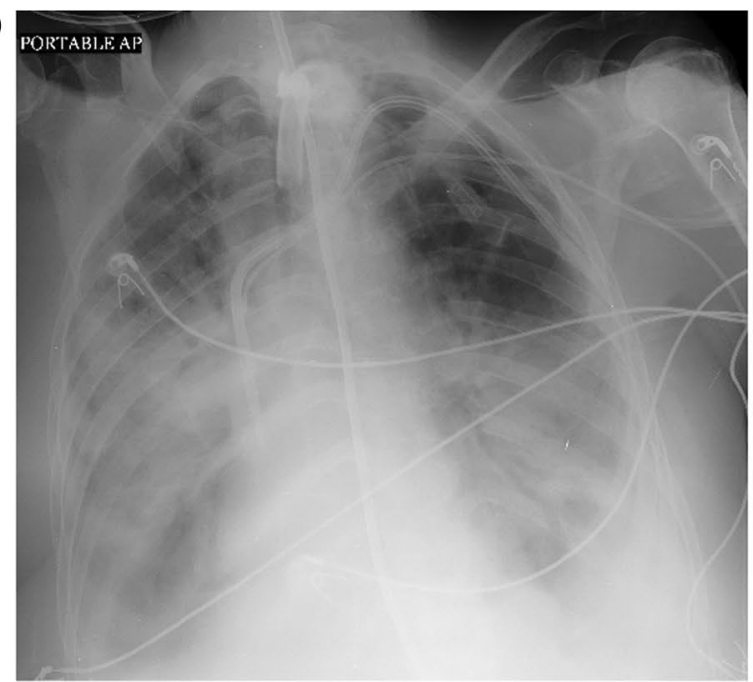

(c)

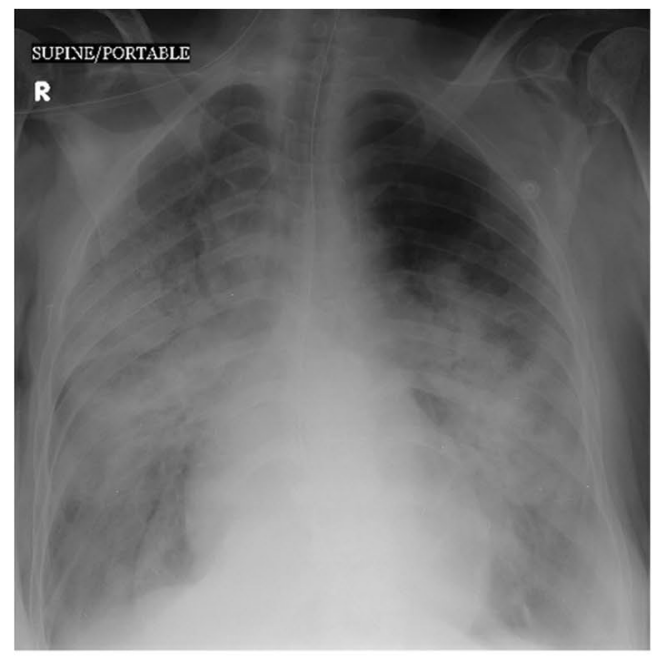

(d)

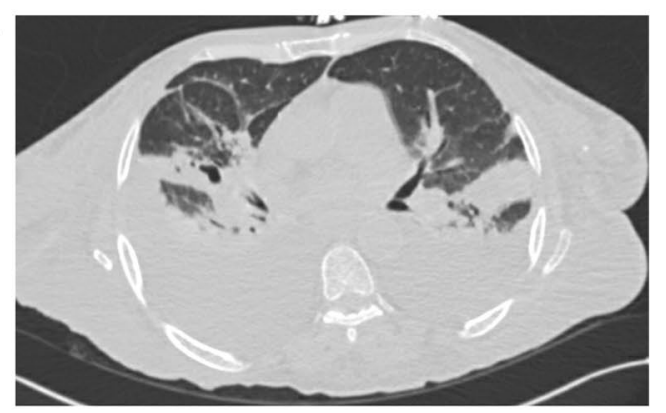


Table 3 Multivariate cox regression analysis to see association of mortality in 57 MERS-Cov patients

\begin{tabular}{llll}
\hline Variable & $\begin{array}{l}\text { Adjusted HR } \\
\text { ratios }\end{array}$ & $\mathbf{9 5 \% ~ C l}$ & $\boldsymbol{p}$-value \\
\hline Age in years & 0.99 & $0.95-1.03$ & 0.6 \\
Ventilation days & 0.84 & $0.76-0.93$ & 0.001 \\
Chest radiographic score & 1.24 & $1.05-1.47$ & 0.01 \\
Diabetes mellitus & 1.01 & $0.24-4.24$ & 0.99 \\
LN (LDH) & 9.91 & $2.44-40.30$ & 0.001 \\
Gender: Male & 0.58 & $0.13-2.66$ & 0.49 \\
\hline
\end{tabular}

between survivors and those who have died. Serum LDH levels $>512$ were $94 \%$ sensitive and $93 \%$ specific for disease-related death in our population. A recent pandemic of SARS-CoV-2, a SARS-CoV-2 virus that resembles MERS-CoV, has also been associated with substantially increased LDH levels [20]. Chang et al. discovered that a serum LDH cut-off value of 359.50 $\mathrm{U} / \mathrm{L}$ accurately predicted SARS-CoV-2 mortality with 93.8\% specificity and $88.2 \%$ accuracy [20]. Higher LDH levels were an independent risk factor for SARSCoV-2 severity (adjusted HR: 2.73, 95\% CI: 1.25-5.97, $p=0.012$ ) and mortality (adjusted HR: 40.50, 95\% CI: $3.65-449.28, p=0.003$ ) according to logistic regression analysis and the Cox proportional hazards model [20]. We do not know why MERS-CoV and SARS-CoV-2 have different ROC values, but we believe MERS-CoV has a greater fatality rate and more severe respiratory symptoms than SARS-CoV-2 [21, 22]. SARS-CoV-2, SARS-CoV-1, and MERS-CoV are linked with a mortality rate of $6.76 \%, 9.6 \%$, and $35.5 \%$, respectively [21, 22].

In our current series, the chest radiography score for the deceased group was considerably higher $(13 \pm 2.6$ 5 vs. $8 \pm 5.6, p=0.001)$ than for the recovered group. Significant inverse relationships between radiography scores and $\mathrm{SaO} 2$ have been reported [7, 23]. All patients with diffuse consolidation required extra ventilation days [7]. We discovered a strong correlation between the chest radiographic score and the number of ventilation days in our current sample $(r=0.44, p=0.001)$.

Although SARS-CoV-2, SARS-CoV-1, and MERS$\mathrm{CoV}$ all frequently infect the liver, various complicating variables may skew the interpretation of existing data about the association between infection and hepatocyte damage [24]. The serum aminotransferases AST and ALT are two of the most critical enzymes for diagnosing liver illness or injury. The AST level is less specific for the liver than for the ALT level. Duan et al. reported that about $38 \%$ of SARS-CoV-1 patients exhibited elevated ALT activity, with higher levels linked to more severe disease [25]. The average increase in ALT and
AST activity was 3 xULN and 1.5 xULN, respectively, in MERS patients [10]. Increased ALT and AST levels were seen in 3xULN and 8xULN of the present cohort of deceased MERS-CoV patients, respectively. We do not know what is driving our population's disproportionate rise in AST. Increased AST levels, but not ALT levels, were significantly higher in people with SARSCoV-2 who had gastrointestinal symptoms than in those who did not (29.35 vs. 24.4, $p=0.02)$ [24]. We did not associate the hematological markers with the clinical presentation of the patients in this group.

Notably, the chest radiographic characteristics of 99 patients were previously reported in four separate studies $[5,26-28]$ focusing solely on the radiological aspects of MERS-CoV pneumonia in chest radiographs, from which 57 cases with LDH levels were selected for the present study. Therefore, the present study examines the relationship between laboratory parameters and chest radiographic score in MERS-CoV pneumonia patients to determine if $\mathrm{LDH}$ is associated with increased mortality risk.

\section{Limitation}

One of the most significant drawbacks of our study was the small sample size. As a result, differentiating between genuine and random effects is difficult. Excluding individuals who do not have LDH findings may add to the bias toward more severe cases. Since the research was retrospective, patients lacked appropriate laboratory measurements and follow-ups. We found death in 18 of 57 patients, and statistical bias may have influenced our results owing to the limited sample size. The hazard ratio's 95 percent confidence interval is higher than the study's inadequate sample size. The research lacks external validation owing to the scarcity of data.

\section{Conclusion}

Like SARS-CoV-1 and SARS-CoV-2, MERS-CoV has pandemic potential and continues to show itself in a people as a sporadic illness. According to our findings, serum LDH levels of $>512$ had a sensitivity of $94 \%$ and a specificity of $93 \%$ for predicting in-hospital mortality in patients infected with MERS-CoV. Diabetes mellitus and a high chest radiographic score were risk factors, suggesting that their presence raises the likelihood that the illness would worsen and aggravate the burden of the pre-existing condition. These objective indicators may identify patients with a good prognosis. These markers may aid in the identification of patients who need additional rigorous therapy. To generalize the findings, a large cohort study with a sufficient sample size may be performed. 


\begin{abstract}
Abbreviations
MERS: Cov-Middle East respiratory syndrome coronavirus; LDH: Lactate dehydrogenase; CAP: Community-acquired pneumonia; ALT: Alanine transaminase; AST: Aspartate aminotransferase; ULN: Upper limit of normal; HR-Hazard Ratio; LN: Log Natural; SARS-CoV-2: Severe acute respiratory syndrome coronavirus 2; SARS-Cov-1: Severe acute respiratory syndrome coronavirus-1; RT-PCR: Reverse transcription Polymerase chain reaction; ARDS: Adult respiratory distress syndrome.
\end{abstract}

\section{Acknowledgements \\ Not applicable}

\section{Authors' contributions}

KMD, JAA, KAD, SS, TA, RS participated in substantial contributions to the conception or design of the work; or the acquisition, analysis, or interpretation of data for the work. KMD, RS, TA and SKO involved in drafting the work or revising it critically for important intellectual content. KMD, JAA, KAD and SKO involved in final approval of the version to be published. KMD contributed to the agreement to be accountable for all aspects of the work in ensuring that questions related to the accuracy or integrity of any part of the work are appropriately investigated and resolved. All authors have read and approved the manuscript.

\section{Funding}

University seed grant was awarded of 40,000 AED for the research. No companies involved. The money was used for the payment of the students for the collection of data.

\section{Availability of data and material}

Yes.

\section{Declarations}

\section{Ethics approval and consent to participate}

Obtained with waiver of the consent form. It is a retrospective analysis of the data. It was issued by the Al Ain Hospital (AAH) Research Ethics committee on 8th Feb 2018 (Ref no AAHEC-02-18-079).

\section{Consent for publication}

Waiver was obtained from the ethical committee.

\section{Competing interests}

The authors declare that they have no competing interests.

\begin{abstract}
Author details
'Department of Radiology, College of Medicine and Health Sciences, UAEU, 17666 Al Ain, United Arab Emirates. ${ }^{2}$ Department of Biostatistics, All India Institute of Medical Sciences, New Delhi, India. ${ }^{3}$ Department of Medical Imaging, King Fahd Medical City, Riyadh, Saudi Arabia. ${ }^{4}$ Department of Physiology, College of Medicine and Health Sciences, UAEU, AI Ain, United Arab Emirates. ${ }^{5}$ Department of Pharmacology, CMHS, UAEU, AI Ain, United Arab Emirates.

${ }^{6}$ Department of Radiology, Tawam Hospital, Al Ain, United Arab Emirates.
\end{abstract}

Received: 6 August 2021 Accepted: 28 September 2021

Published online: 05 October 2021

\section{References}

1. Perlman S, Zumla A (2021) MERS-CoV in Africa-an enigma with relevance to COVID-19. Lancet Infect Dis 21:303-305

2. Memish ZA, Perlman S, Van Kerkhove MD, Zumla A (2020) Middle East respiratory syndrome. The Lancet 395:1063-1077

3. Control ECfDPa (5 March, 2021) Geographical distribution of confirmed MERS-CoV cases by country of infection and year, ECDC. https://www. ecdc.europa.eu/en/publications-data/geographical-distribution-confi rmed-mers-cov-cases-country-infection-and-year

4. Baharoon S, Memish ZA (2019) MERS-CoV as an emerging respiratory illness: a review of prevention methods. Travel Med Infect Disease 32:101520
5. Das KM, Lee EY, Al Jawder SE, Enani MA, Singh R, Skakni L, Al-Nakshabandi N, AlDossari K, Larsson SG (2015) Acute Middle East Respiratory syndrome coronavirus: temporal lung changes observed on the chest radiographs of 55 patients. AJR Am J Roentgenol 205:W267-274. https://doi.org/10. 2214/ajr.15.14445

6. Ooi GC, Daqing M (2003) SARS: radiological features. Respirology 8:S15-S19

7. Ooi CGC, Khong PL, Ho JCM, Lam B, Wong WM, Yiu WC, Wong PC, Wong CF, Lai KN, Tsang KWT (2003) Severe acute respiratory syndrome: radiographic evaluation and clinical outcome measures. Radiology 229:500-506

8. Arabi YM, Arifi AA, Balkhy HH, Najm H, Aldawood AS, Ghabashi A, Hawa $H$, Alothman A, Khaldi A, Al Raiy B (2014) Clinical course and outcomes of critically ill patients with Middle East respiratory syndrome coronavirus infection. Ann Intern Med 160:389-397

9. Alfaraj SH, Al-Tawfiq JA, Assiri AY, Alzahrani NA, Alanazi AA, Memish ZA (2019) Clinical predictors of mortality of Middle East Respiratory Syndrome Coronavirus (MERS-CoV) infection: a cohort study. Travel Med Infect Dis 29:48-50

10. Saad M, Omrani AS, Baig K, Bahloul A, Elzein F, Matin MA, Selim MAA, Al Mutairi M, Al Nakhli D, Al Aidaroos AY (2014) Clinical aspects and outcomes of 70 patients with Middle East respiratory syndrome coronavirus infection: a single-center experience in Saudi Arabia. Int J Infect Dis 29:301-306

11. Drent M, Cobben NA, Henderson RF, Wouters EF, van Dieijen-Visser M (1996) Usefulness of lactate dehydrogenase and its isoenzymes as indicators of lung damage or inflammation. Eur Respir J 9:1736-1742

12. Huijgen HJ, Sanders GTB, Koster RW, Vreeken J, Bossuyt PMM (1997) The clinical value of lactate dehydrogenase in serum: a quantitative review. Eur J Clin Chem Clin Biochem 35:569-580

13. Terpstra ML, Aman J, van Nieuw Amerongen GP, Groeneveld ABJ (2014) Plasma biomarkers for acute respiratory distress syndrome: a systematic review and meta-analysis. Crit Care Med 42:691-700

14. Das KM, Lee EY, Singh R, Enani MA, Al Dossari K, Van Gorkom K, Larsson SG, Langer RD (2017) Follow-up chest radiographic findings in patients with MERS-CoV after recovery. Indian J Radiol Imaging 27:342

15. Assiri A, Al-Tawfiq JA, Al-Rabeeah AA, Al-Rabiah FA, Al-Hajjar S, Al-Barrak A, Flemban H, Al-Nassir WN, Balkhy HH, Al-Hakeem RF (2013) Epidemiological, demographic, and clinical characteristics of 47 cases of Middle East respiratory syndrome coronavirus disease from Saudi Arabia: a descriptive study. Lancet Infect Dis 13:752-761

16. The WHO MERS-CoV Research Group (2013) State of knowledge and data gaps of Middle East respiratory syndrome coronavirus (MERS-CoV) in humans. PLoS Curr. https://doi.org/10.1371/currents.outbreaks.0bf71 9e352e7478f8ad85fa30127ddb8

17. Rivers CM, Majumder MS, Lofgren ET (2016) Risks of death and severe disease in patients with Middle East respiratory syndrome coronavirus, 2012-2015. Am J Epidemiol 184:460-464

18. Ahmed AE (2017) The predictors of 3-and 30-day mortality in 660 MERSCoV patients. BMC Infect Dis 17:1-8

19. Al Ghamdi M, Alghamdi KM, Ghandoora Y, Alzahrani A, Salah F, Alsulami A, Bawayan MF, Vaidya D, Perl TM, Sood G (2016) Treatment outcomes for patients with Middle Eastern Respiratory Syndrome Coronavirus (MERS CoV) infection at a coronavirus referral center in the Kingdom of Saudi Arabia. BMC Infect Dis 16:1-7

20. Li C, Ye J, Chen Q, Hu W, Wang L, Fan Y, Lu Z, Chen J, Chen Z, Chen S (2020) Elevated lactate dehydrogenase (LDH) level as an independent risk factor for the severity and mortality of COVID-19. Aging (Albany NY) 12:15670

21. Lu L, Zhong W, Bian Z, Li Z, Zhang K, Liang B, Zhong Y, Hu M, Lin L, Liu J (2020) A comparison of mortality-related risk factors of COVID-19, SARS, and MERS: a systematic review and meta-analysis. J Infect 81:e18-e25

22. World Health Organization (2020) World Health Organization coronavirus disease 2019 (COVID-19) situation report

23. Ho JC, Ooi GC, Mok TY, Chan JW, Hung I, Lam B, Wong PC, Li PC, Ho PL, Lam WK (2003) High-dose pulse versus nonpulse corticosteroid regimens in severe acute respiratory syndrome. Am J Respir Crit Care Med 168:1449-1456

24. Kukla M, Skonieczna-Żydecka K, Kotfis K, Maciejewska D, Łoniewski I, Lara L, Pazgan-Simon M, Stachowska E, Kaczmarczyk M, Koulaouzidis A (2020) 
COVID-19, MERS and SARS with concomitant liver injury—systematic review of the existing literature. J Clin Med 9:1420

25. Duan ZP, Chen Y, Zhang J, Zhao J, Lang ZW, Meng FK, Bao XL (2003) Clinical characteristics and mechanism of liver injury in patients with severe acute respiratory syndrome. Chin J Hepatol 11:493-496

26. Das KM, Lee EY, Enani MA, AlJawder SE, Singh R, Bashir S, Al-Nakshbandi N, AlDossari K, Larsson SG (2015) CT correlation with outcomes in 15 patients with acute Middle East respiratory syndrome coronavirus. Am J Roentgenol 204:736-742

27. Das KM, Alkoteesh JA, Sheek-Hussein M, Alzadjali SA, Alafeefi MT, Singh R, Statsenko Y, Soteriades ES, Singh V, Gorkom KV (2021) Role of chest radiograph in MERS-Cov pneumonia. A single tertiary referral center experience in the United Arab Emirates. Egypt J Radiol Nucl Med 52(1):1-7

28. Das KM, Lee EY, Langer RD, Larsson SG (2016) Middle East Respiratory syndrome coronavirus: what does a radiologist need to know? AJR Am J Roentgenol 206:1193-1201. https://doi.org/10.2214/ajr.15.15363

\section{Publisher's Note}

Springer Nature remains neutral with regard to jurisdictional claims in published maps and institutional affiliations.

\section{Submit your manuscript to a SpringerOpen ${ }^{\circ}$ journal and benefit from:}

- Convenient online submission

- Rigorous peer review

- Open access: articles freely available online

- High visibility within the field

- Retaining the copyright to your article

Submit your next manuscript at $\boldsymbol{\nabla}$ springeropen.com 